Louisiana State University

LSU Digital Commons

Faculty Publications

Department of Physics \& Astronomy

8-6-2009

\title{
Subcycle spatial mapping of recollision dynamics
}

\author{
N. Dudovich \\ Weizmann Institute of Science Israel \\ J. L. Tate \\ Louisiana State University \\ Y. Mairesse \\ National Research Council Canada \\ D. M. Villeneuve \\ National Research Council Canada \\ P. B. Corkum \\ National Research Council Canada
}

See next page for additional authors

Follow this and additional works at: https://digitalcommons.Isu.edu/physics_astronomy_pubs

\section{Recommended Citation}

Dudovich, N., Tate, J., Mairesse, Y., Villeneuve, D., Corkum, P., \& Gaarde, M. (2009). Subcycle spatial mapping of recollision dynamics. Physical Review A - Atomic, Molecular, and Optical Physics, 80 (1) https://doi.org/10.1103/PhysRevA.80.011806

This Article is brought to you for free and open access by the Department of Physics \& Astronomy at LSU Digital Commons. It has been accepted for inclusion in Faculty Publications by an authorized administrator of LSU Digital Commons. For more information, please contact ir@lsu.edu. 


\section{Authors}

N. Dudovich, J. L. Tate, Y. Mairesse, D. M. Villeneuve, P. B. Corkum, and M. B. Gaarde 


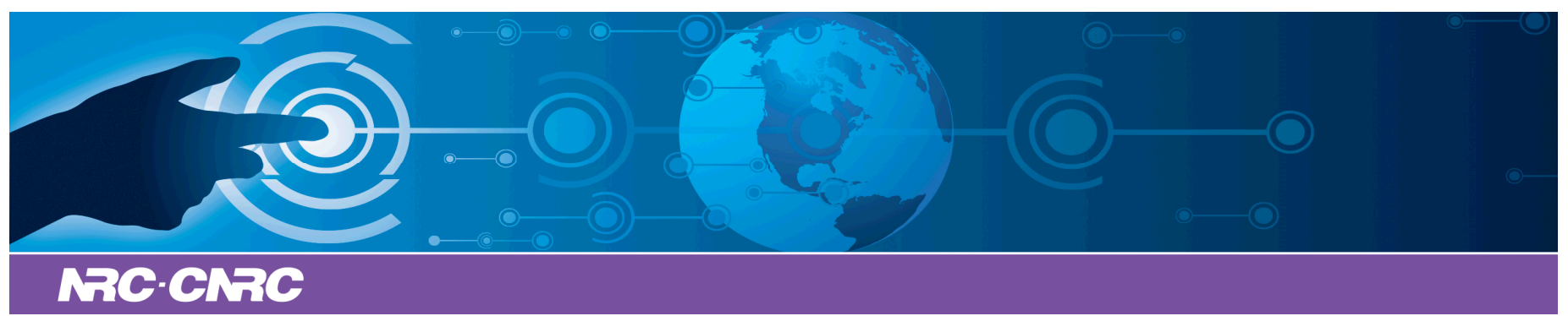

\title{
NRC Publications Archive Archives des publications du CNRC
}

\author{
Subcycle spatial mapping of recollision dynamics \\ Dudovich, N.; Tate, J. L.; Mairesse, Y.; Villeneuve, D. M.; Corkum, P. B.; \\ Gaarde, M. B.
}

This publication could be one of several versions: author's original, accepted manuscript or the publisher's version. / La version de cette publication peut être l'une des suivantes : la version prépublication de l'auteur, la version acceptée du manuscrit ou la version de l'éditeur.

For the publisher's version, please access the DOI link below./ Pour consulter la version de l'éditeur, utilisez le lien DOI ci-dessous.

\section{Publisher's version I Version de l'éditeur:}

https://doi.org/10.1103/PhysRevA.80.011806

Physical Review A, 80, pp. 011806-1-011806-4, 2009-07-01

\section{NRC Publications Record / Notice d'Archives des publications de CNRC:}

https://nrc-publications.canada.ca/eng/view/object/?id=15a4d6c5-70c5-442e-a890-662af1eead51 https://publications-cnrc.canada.ca/fra/voir/objet/?id=15a4d6c5-70c5-442e-a890-662af1eead51

Access and use of this website and the material on it are subject to the Terms and Conditions set forth at https://nrc-publications.canada.ca/eng/copyright

READ THESE TERMS AND CONDITIONS CAREFULLY BEFORE USING THIS WEBSITE.

L'accès à ce site Web et l'utilisation de son contenu sont assujettis aux conditions présentées dans le site https://publications-cnrc.canada.ca/fra/droits

LISEZ CES CONDITIONS ATTENTIVEMENT AVANT D'UTILISER CE SITE WEB.

Questions? Contact the NRC Publications Archive team at

PublicationsArchive-ArchivesPublications@nrc-cnrc.gc.ca. If you wish to email the authors directly, please see the first page of the publication for their contact information.

Vous avez des questions? Nous pouvons vous aider. Pour communiquer directement avec un auteur, consultez la première page de la revue dans laquelle son article a été publié afin de trouver ses coordonnées. Si vous n'arrivez pas à les repérer, communiquez avec nous à PublicationsArchive-ArchivesPublications@nrc-cnrc.gc.ca. 


\title{
Subcycle spatial mapping of recollision dynamics
}

\author{
N. Dudovich, ${ }^{1,2}$ J. L. Tate, ${ }^{3}$ Y. Mairesse, ${ }^{2,4}$ D. M. Villeneuve, ${ }^{2}$ P. B. Corkum, ${ }^{2}$ and M. B. Gaarde ${ }^{3}$ \\ ${ }^{1}$ Department of Physics of Complex Systems, Weizmann Institute of Science, Rehovot 76100, Israel \\ ${ }^{2}$ National Research Council of Canada, 100 Sussex Drive, Ottawa, Ontario, Canada K1A OR6 \\ ${ }^{3}$ Department of Physics and Astronomy, Louisiana State University, Baton Rouge, Louisiana 70803-4001, USA \\ ${ }^{4}$ CELIA, Université Bordeaux I, UMR 5107, 351 Cours de la Libération, 33405 Talence Cedex, France
}

(Received 27 August 2008; published 21 July 2009)

\begin{abstract}
We present a method for controlling the spatial properties of a high-order harmonic beam on a subcycle time scale. By adding a second-harmonic field to the driving laser field, we modify the spatiotemporal structure of the harmonic beam and manipulate it with attosecond resolution. Such a manipulation maps the subcycle dynamics of a recolliding electron to the spatial domain.
\end{abstract}

DOI: $10.1103 /$ PhysRevA.80.011806

PACS number(s): 42.65.Ky, 42.65.Re

Time-space coupling is commonly used in third-order processes to generate and manipulate femtosecond pulses [1]. This coupling is also applied to map weak temporal variations to the spatial domain and therefore to enhance the sensitivity of the nonlinear measurements by orders of magnitude [2]. One example is the measurement of femtosecond dynamics in solids, liquids, or gases via transient grating spectroscopy, in which weak temporal information is transferred into the spatial domain where it is measured against zero background [3]. Extending this approach into the evolving field of attosecond science will play an important role in a broad range of experiments.

With the advent of attosecond technology, time-resolved studies of the ultrafast dynamics of bound electrons have become feasible [4]. Attosecond extreme ultraviolet (XUV) pulses can be produced during the interaction between a strong laser field and a gas of atoms. In each optical cycle of the laser field an electron is removed from the atom, accelerated by the strong laser field, and recollides with the parent ion $[5,6]$. There are two approaches to perform time-resolved measurements with attosecond resolution. In one approach the attosecond pulse is used to pump a fast dynamic process [4]. In the other approach the ionized electron is utilized as an ultrafast probe to measure molecular dynamics [7-10]. Recently a significant breakthrough in the study of high harmonic generation (HHG) processes demonstrated that the recollision electron can probe different molecular orbitals. This advance allows the observation of internal dynamics in molecular systems. McFarland et al. [11] showed that high harmonics in $\mathrm{N}_{2}$ molecules are generated by the interplay between the highest occupied molecular orbital (HOMO) and the HOMO-1 orbital. Smirnova et al. [12] demonstrated that tunnel ionization in $\mathrm{CO}_{2}$ molecules launches a wave packet composed of the HOMO and HOMO-2 orbitals, which evolves during the optical cycle. Li et al. [13] showed that the HOMO orbital switches during a molecular vibration of $\mathrm{NO}_{2}$ dimers. These studies open the door to the observation of internal electronic processes in molecules.

Common to these studies is the extraction of temporal information from the harmonic spectrum, in which the freeelectron dynamics, the structure of the orbitals and their temporal evolution are all imprinted. In small molecules the observation of the different orbitals can be achieved in a simple manner by scanning a single parameter of the light matter interaction such as the alignment angle [11], the bond length [13], or the laser intensity [12]. However, internal electronic processes in complex molecules involve a large number of degrees of freedom which leads us to ask whether we can decouple the free-electron dynamics from the different orbitals and resolve them independently. Decoupling the numerous degrees of freedom requires increasing the dimensionality and the contrast of the measurement.

In this Rapid Communication we introduce an additional domain in the measurement of harmonic spectra via the macroscopic coupling between space and time. Spatiotemporal coupling in high harmonic generation has been shown to offer an important degree of control over the coherence properties of the harmonic radiation [14-16]. Recently it has been demonstrated that HHG produced by a grating of rotationally excited molecules probe the excitation with notably high contrast [17]. Here we introduce the concept of how one can engineer the space-time coupling in the strong field recollision process, in order to utilize the spatial domain in reconstruction of bound-state dynamics from harmonic spectra. We show both experimental and theoretical results supporting our predictions.

We couple space and time by manipulating the recollision process, adding a somewhat weaker second-harmonic (blue) field to the strong infrared (IR) laser field and controlling the relative delay between the two pulses and the relative size of the two beams. By changing the recollision time across the near field spatial distribution of a harmonic, we alter its phase front, and this is imprinted on the far-field spatial profile. The manipulation of the electron recollision dynamics therefore constitutes an induced optical element [18]. In our case the dynamics is dominated by the recollision process. However, if the initial state changes between the time of ionization and recollision, this dynamics will also dramatically affect both the shape and the temporal properties of the induced element. Thus, attosecond dynamics will be imprinted on the far-field distribution of the high harmonics and can be resolved with subcycle accuracy and very high contrast. As a specific example, we will discuss ground-state dynamics in $\mathrm{CO}_{2}$ molecules in the conclusion part of this Rapid Communication.

We introduce the basic mechanism of our approach by first analyzing the single atom response to the two-color field and then extending the analysis to include macroscopic ef- 


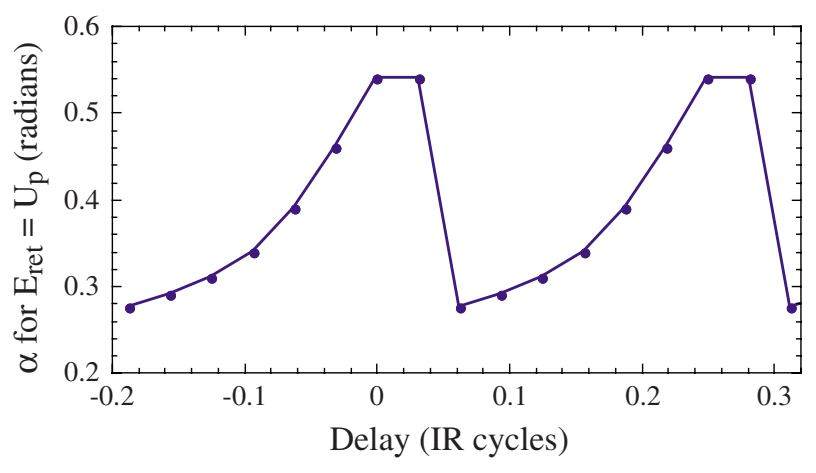

FIG. 1. (Color online) Phase coefficient $\alpha$ as a function of the red-blue delay for short-path trajectories which give rise to return energies around $1 U_{p}$, from the dominant family of trajectories. The IR-alone value for $\alpha$ around $1 U_{p}$ is $0.4 \mathrm{rad}$.

fects. In our experiment, the intensity of the blue light is $10 \%$ of the IR intensity. This means that the two-color electric field is strongly asymmetric in time with one half cycle being much stronger than the other for most delays. For any given delay we can identify two families of electron trajectories in each calculation, each with short and long trajectories that are released in the two different half cycles of the field [19]. The complete harmonic spectrum generated by the two-color field consists of the coherent sum of the contributions from both families. However, for most delays the spectrum is strongly dominated by the family in which electrons are released in the stronger of the two half cycles and are reaccelerated back to the ion cores in the weaker half cycle [19]. In the following we consider the influence of the red-blue delay on the electron dynamics for the short trajectory in the dominant family of trajectories [20].

In a one-color field, the short trajectory contribution to a high-order harmonic is characterized by its intensity dependent phase $\phi=\alpha U_{p} / \omega$, where $U_{p} / \omega$ is the ponderomotive energy measured in units of the laser photon energy. The phase coefficient $\alpha$ is directly related to the electron's travel time [21]. For a bichromatic field with a given delay $t_{d}$ and a given red-blue intensity ratio, we calculate the phase coefficient $\alpha\left(t_{d}\right)$ via the quasiclassical action as outlined in [21] for a monochromatic field. As is the case for a one-color field, $\alpha\left(t_{d}\right)$ varies with return energy. We concentrate here on a return energy of $E_{\mathrm{ret}}=1 U_{p}$, corresponding to harmonics in the low-plateau range of the spectrum. Figure 1 shows the variation in $\alpha\left(t_{d}\right)$ with delay. The figure shows that $\alpha$ varies by a factor of 2 and that the variation is asymmetric: there is a slow increase starting around $-0.2 T_{1}$ until about 0 , followed by a sharp decrease, where $T_{1}$ is the fundamental field period. The two families give rise to similar cut-off energies and similar yields around $0.05 T_{1}$, at which point the other family becomes dominant and the delay dependence is repeated. The change in $\alpha$ with delays is due to the change in the electron's travel time imposed by the second field. The travel time can be either shorter or longer than in the IR alone case, yielding a phase coefficient which is smaller or larger than the IR-alone value of $0.4 \mathrm{rad}$.

The macroscopic consequences of the delay dependence demonstrated in Fig. 1 can be appreciated by considering the simplest possible case of the two beams having the same focal spot size so that their intensity ratio stays constant across the focus. Since the intensity dependent phase is proportional to $\alpha$, Fig. 1 predicts that as we change the delay from $-0.2 T_{1}$ toward 0 we will increase the curvature of the harmonic phase front across the laser focus and therefore impose a larger divergence on the harmonic beam. Because of the large change in $\alpha$ with delay, this manipulation will lead to a significant variation in the harmonic divergence, acting as an effective positive or negative lens compared to the IR-alone case.

Now we turn to experiment. We demonstrate subcycle spatial control by generating high harmonics in argon with a two-color field. The $800 \mathrm{~nm} 50 \mathrm{~Hz}$ fundamental pulse has a duration of $30 \mathrm{fs}$. The second-harmonic field is produced using a $\mathrm{BaB}_{2} \mathrm{O}_{4}(\mathrm{BBO})$ crystal placed before the focusing mirror and the conversion efficiency is about $2 \%$. The relative phase of the IR and the blue fields is controlled with a piece of glass. Group-velocity dispersion is compensated using a birefringent crystal (calcite). To obtain two parallel polarized fields, a zero-order wave plate rotates the fundamental and the second-harmonic fields by $90^{\circ}$ and $180^{\circ}$, respectively (see Ref. [22]). The BBO aperture is smaller than the IR beam size, therefore the second-harmonic field is generated by an almost uniform IR field. As a result, the two colors have approximately the same beam diameters before the focusing mirror. The IR beam is focused at approximately $3 \times 10^{14} \mathrm{~W} / \mathrm{cm}^{2}$ in the jet with a focal spot of $\sim 100 \mu \mathrm{m}$, while the blue focus is about half the size of the IR focus which gives an intensity ratio of $\sim 0.1$. The IR is focused a few $\mathrm{mm}$ before the center of the jet. The gas jet length is about $500 \mu \mathrm{m}$, and the gas pressure is a few torrs. The harmonic spectrum is measured by an XUV spectrometer.

Figures 2(a)-2(c) shows the far-field distribution of harmonics 18,21 , and 24. As we scan the delay, we observe strong modulations of the far-field profile. In the figure we observe an arrow like structure as the spatial profile slowly broadens with delay from a narrow collimated structure to an annular beam and then abruptly changes back to the narrow structure. At the delays where the divergence is maximized we observe a contribution of both off-axis and on-axis radiations. Odd and even harmonics are modulated in phase, but the contrast is in general lower for odd than for even harmonics.

The experimental results shown in Fig. 2 can be interpreted as a direct mapping of a subcycle temporal manipulation of the electron dynamics controlled by the strength and phase of the blue field into the macroscopic spatial domain as predicted by our simple model discussed in connection with Fig. 1. Figure 2 also shows a comparison to complete calculations of the macroscopic harmonic response to the two-color electric field distributions via the coupled solutions of the Maxwell wave equation and the time-dependent Schrödinger equation. Details on our approach can be found in [23]. In the calculations we have chosen parameters to closely match the experimental ones, in terms of the pulse durations and peak intensities, the focusing conditions of the two beams, the length, and pressure of the argon gas jet. The blue focus $(40 \mu \mathrm{m})$ is thus approximately half the size of the IR focus $(90 \mu \mathrm{m})$. In Figs. 2(d)-2(f) we show the calculated 


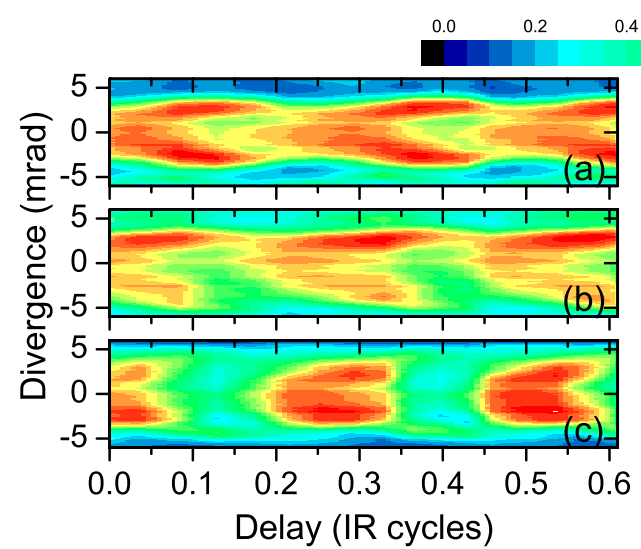

far-field spatial profiles of harmonics 18, 21, and 24. The qualitative agreement between the theoretical and the experimental results is very good-the spatial profiles change dramatically with delay, forming the same arrowlike structure in the figure, and the modulation of the odd harmonics is reduced compared to the even harmonics. The divergence also increases with harmonic order due to the increase in $\alpha$ with return energy. Quantitatively, the theoretical far-field profiles are somewhat narrower than the experimental ones although the changes in divergence with delay are similar.

In the experiment and calculations shown in Fig. 2 we have achieved additional control over the harmonic divergence as compared to the simple prediction in Fig. 1 by allowing the two beams to have different focal waists. This means we can tune both their relative strengths and phases across the beam profile. The additional control is illustrated in Fig. 3, which presents the result of macroscopic calculations as described above. The figure shows the radial phase variation in the 19th harmonic at the end of a low-pressure $0.5 \mathrm{~mm}$ long argon gas jet for delay of $t_{d}=0$ [Fig. 3(a) ] and $t_{d}=0.125 T_{1}$ [Fig. 3(b)]. For clarity we show only the short trajectory contribution to the 19th harmonic. For both delays, we compare the radial phase variation caused by a $90 \mu \mathrm{m}$ IR beam alone to the phases caused by the IR beam combined with a small blue beam $(40 \mu \mathrm{m})$ or with a large blue beam $(90 \mu \mathrm{m})$. The size of the blue beam clearly offers additional control of the harmonic phase front. When the blue beam is small, for instance, the harmonic beam has an inner region dominated by red-blue dynamics and an outer region dominated by IR-alone dynamics. The smaller size of the blue beam also explains why the odd harmonics exhibit a reduced modulation of their spatial profile compared to the even harmonics. The blue beam induces an effective aperture in the near field. The even harmonics are only generated where the blue light overlaps the IR light and breaks the half-cycle periodicity of the IR field. The generation of odd harmonics does not require such an overlap. However, since the blue light is strong enough, odd harmonics are modulated as well and experience the blue beam as an effective aperture.

The modification of the far-field profile with the red-blue delay indicates that the induced optical element changes within the optical cycle. Our theoretical analysis allows us to resolve the time dependence of the basic mechanism. We separate the two families of electron trajectories to be initiated-one when the electric field is positive, the other when it is negative [19]. The resulting spatial profiles for the harmonic 20 are shown in Figs. 4(a) and 4(b). Figure 4(c) shows the full calculation, corresponding to the coherent sum of the two separate contributions. Comparing Figs. 4(a)-4(c) we can follow the temporal evolution of the beam profile. The negative family dominates the harmonic emission at delays between $0.075 T_{1}$ and $0.3 T_{1}$. Starting at $0.3 T_{1}$, the contribution of the positive family becomes significant, leading to a far field composed of both a narrow beam and an annular beam. At delays larger than $0.35 T_{1}$, the negative family no longer contributes and the spatial profile is dominated by the narrow beam due to the positive family. This analysis clearly shows that the spatial properties of the beam change within the optical cycle and can be accurately manipulated by the two field delays. If we focus on delays around $0.3 T_{1}$, where the two families contribute equally, we observe a rapid variation in the beam divergence with delay. The beam profile changes from a narrow shape to a ring shape within a few hundred attoseconds.

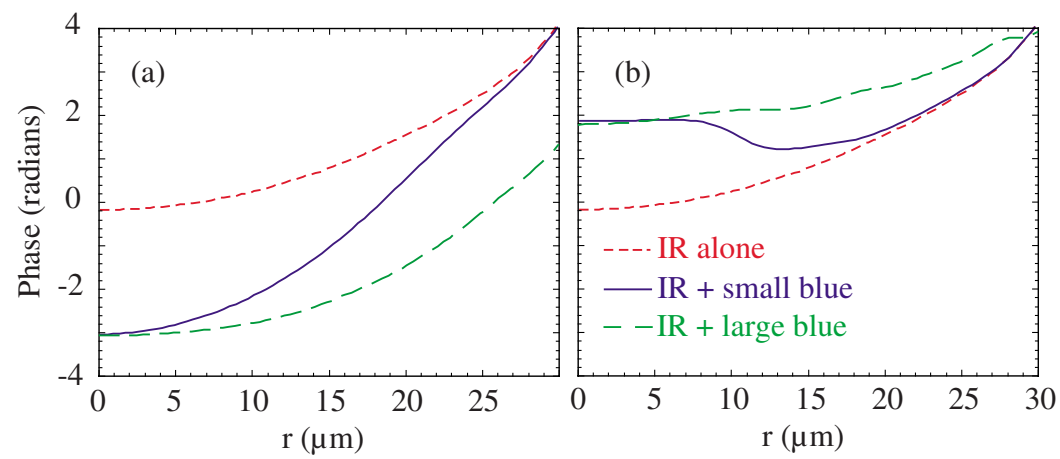

FIG. 3. (Color online) The calculated radial phase variation in the short-path contribution to harmonic 19 at the end of the argon gas [20]. The calculation is performed for two different delays: (a) $t_{d}=0$ and (b) $t_{d}=0.125 T_{1}$. 


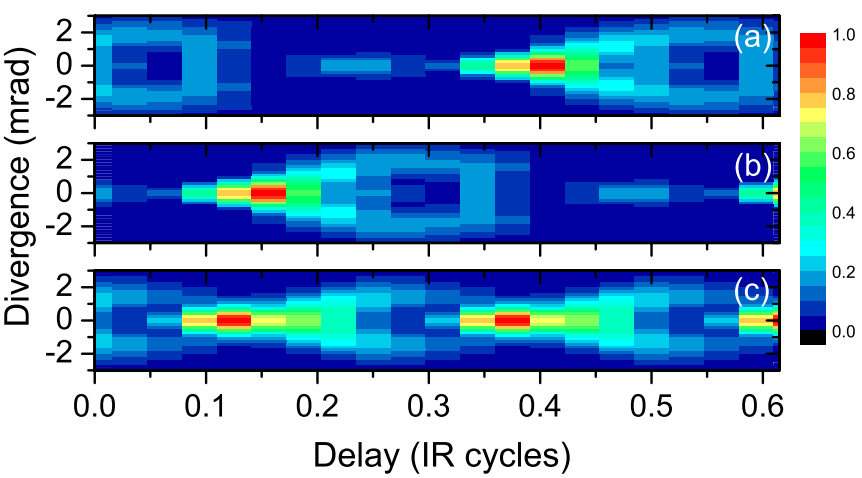

FIG. 4. (Color online) [(a) and (b)] Separate contributions to harmonic 20 from trajectories initiated during (a) positive or (b) negative half-cycles of the two-color field, (c) shows the full calculation.

In this Rapid Communication we have focused on the manipulation of the free-electron dynamics. However, our approach can be applied to map the ground-state dynamics into the far-field distribution as well. There are two main advantages proposed in this Rapid Communication, compared to previous experiments which utilize the recolliding electron as a fast probed. The first is the probing applied by the recolliding electron. Using a two-color scheme we can accurately tune the trajectory length and therefore the probing time. The second is the reading - using the spatiotemporal coupling we read the probing with very high contrast.

In the following we describe a proposed scheme to measure inner-orbital dynamics in $\mathrm{CO}_{2}$ molecules. Tunnel ion- ization in $\mathrm{CO}_{2}$ molecules launches a wave packet composed of the HOMO and HOMO-2 orbitals which evolves during the optical cycle. Destructive interference between the two orbitals occurs when their phase difference reaches the value of $\pi$, leading to a minimum observed in the HHG spectrum [12]. Scanning the two-color delay will change the electron trajectories - the probing time-and therefore will modify the timing of the destructive interference and the position of the minima in the HHG spectrum. Adding the macroscopic scale where electron trajectories are naturally scanned across the beam's profile, the minima will be located at a specific distance from the center of the beam leading to a clear diffraction pattern at the far field. The diffraction pattern would allow us to resolve the hole dynamics with notably high contrast.

As in many spatiotemporal schemes, mapping the dynamics to the far-field profile improves the contrast of the measurement by orders of magnitude. Such schemes, applied in the picosecond and femtosecond regimes have become the method of choice to resolve molecular dynamics. We expect that its extension into the attosecond regime will play an important role in resolving electron's dynamic.

We acknowledge partial financial support by the National Research Council of Canada, the Canadian Institute for Photonic Innovation, NSERC Accelerator grant program, and NSERC Special Research Opportunities grant program. This material is based upon work supported by the National Science Foundation through Grant No. PHY-0449235 and through the Center for Computation and Technology at Louisiana State University.
[1] Nonlinear Optics, edited by R. W. Boyd (Academic, New York, 1992).

[2] S. Mukamel, Principles of Nonlinear Optical Spectroscopy (Oxford University Press, New York, 1995).

[3] H. J. Eichler, P. Gunther, and D. W. Pohl, Laser-induced Dynamic Gratings (Springer-Verlag, Berlin, 1986).

[4] M. Drescher et al., Nature (London) 419, 803 (2002).

[5] P. B. Corkum, Phys. Rev. Lett. 71, 1994 (1993).

[6] K. J. Schafer, B. Yang, L. F. DiMauro, and K. C. Kulander, Phys. Rev. Lett. 70, 1599 (1993).

[7] H. Niikura et al., Nature (London) 421, 826 (2003).

[8] X. M. Tong, Z. X. Zhao, and C. D. Lin, Phys. Rev. Lett. 91, 233203 (2003).

[9] H. Niikura, D. M. Villeneuve, and P. B. Corkum, Phys. Rev. Lett. 94, 083003 (2005).

[10] S. S. Baker et al., Science 312, 424 (2006).

[11] B. K. McFarland, J. P. Farrell, P. H. Bucksbaum, and M. Guhr, Science 322, 1232 (2008)

[12] O. Smirnova et al., Nature (to be published).
[13] W. Li et al., Science 322, 1207 (2008).

[14] P. Salières, A. L'Huillier, and M. Lewenstein, Phys. Rev. Lett. 74, 3776 (1995).

[15] P. Antoine, A. L'Huillier, and M. Lewenstein, Phys. Rev. Lett. 77, 1234 (1996).

[16] M. Bellini et al., Phys. Rev. Lett. 81, 297 (1998).

[17] Y. Mairesse et al., Phys. Rev. Lett. 100, 143903 (2008).

[18] Y. Mairesse et al., Opt. Lett. 32, 436 (2007).

[19] J. Mauritsson et al., Phys. Rev. Lett. 97, 013001 (2006).

[20] Phase matching conditions are such that harmonics are strongly dominated by the short trajectory contributions. The long trajectory contributions to Figs. 2 and 4 can only be seen on a logarithmic scale and are found much further off axis than what is included in the figures.

[21] M. Lewenstein, P. Balcou, M. Y. Ivanov, A. LHuillier, and P. B. Corkum, Phys. Rev. A 49, 2117 (1994).

[22] N. Dudovich et al., Nat. Phys. 2, 781 (2006).

[23] M. B. Gaarde, J. L. Tate, and K. J. Schafer, J. Phys. B 41, 132001 (2008). 